\title{
Reforma y modernización administrativas. Especial referencia a la Comunidad Autónoma de Galicia
}

\section{J. Vilas Nogueira * y Miguel A. Bastos Boubeta **}

\section{Delimitación conceptual de reforma y modernización}

Aunque no siempre es reconocido por la bibliografía, los procesos de cambio, real o aparente, que se desarrollan en el seno de las Administraciones públicas "occidentales" responden a muy diversas motivaciones y ofrecen tales variaciones, en dirección y énfasis, que hacen muy difícil un enfoque uniforme. El estudio de las "reformas" constituye en estos momentos una de las ramas más fértiles de las publicaciones sobre la Administración, debido a la gran cantidad de "reformas administrativas" emprendidas desde los años 60 (CAIDEN, 1991a: vii) y a la no despreciable cantidad de recursos materiales puestos a disposición de los expertos por los gobiernos.

Las palabras utilizadas para designar aquellos procesos de cambio varian notablemente según los paises, y, en el nuestro, su significado depende frecuentemente del que le hayan querido atribuir los traductores de obras extranjeras (o las editoriales que las han publicado) '. En el plano desiderativo, creemos que deberían distinguirse las modificaciones en la actuación administrativa derivadas de la adaptación de las Administraciones públicas, como reacción a cambios en su entorno, social, económico o político, que llamaremos modernización administrati$\mathrm{va}^{2}$, de aquellos otros procesos que suponen un intento deliberado de transformación, no meramente reactivo al entorno, sino con pretensión de incidir en él ${ }^{3}$, ya sea mediante la mejora de los métodos y técnicas de funcionamiento en gene- ral, ya mediante la mejora del desempeño de los empleados públicos en particular, que llamaremos reforma, en sentido estricto. Naturalmente, la distinción entre acción meramente reactiva y acción transformadora es relativa; en casos extremos, la frontera puede ser muy sutil; y pueden darse transiciones de una a otra, pero siempre pueden separarse conceptualmente.

En este sentido, la modernización administrativa, por su propia naturaleza y a diferencia de las reformas, que tienen un alcance y persiguen objetivos definidos, es un proceso abierto, un proceso sin fin, de reajuste de las estructuras y, sobre todo, de los procesos administrativos, por lo que no puede afirmarse en ningún momento que ha llegado a su fin (NiETO, 1989) ni delimitarse claramente un contenido específico (Trosa, 1992). Así lo entendemos nosotros, aunque la circunstancia de que "modernización" es (por lo menos, entre nosotros) una palabra mucho más "cargadan afectivamente (en sentido positivo) que "reforma" explica su amplia difusión para designar lo que debería ser llamado «reforman.

Los sistemas administrativos occidentales, aun manifestando rasgos comunes susceptibles de singularizarlos respecto de modelos administrativos de otras culturas, como la diferenciación formal entre instancias administrativas y políticas, con subordinación de las primeras a las segundas, por un lado, y la diferenciación entre instancias administrativas y económicas, por otro, presentan modelos diversos entre sí. Por un lado, se puede distinguir un modelo americano de Administración Pública, que ofrece un sistema de reclutamiento de personal dependiente de los procesos electorales políticos, con unas estructuras de carrera y salariales muy diversificadas y flexibles, en el que es osten- 
sible la vinculación a grupos de interés, y un modelo europeo de Administración Pública (PETERS, 1991), con una estricta y homogénea regulación normativa, estructuras de carrera y salariales rígidas y uniformes, garantías legales de permanencia de los funcionarios en sus puestos, y una tradición formal ${ }^{4}$ de ausencia de participación política directa de los funcionarios, en tanto tales.

Podría esperarse que la existencia de estos dos mundos administrativos se proyectase en los tipos de reforma administrativa. Sin embargo, predominan los rasgos y objetivos comunes, entre otras cosas por el enorme grado de difusión internacional que han alcanziado las reformas administrativas acometidas, 0 meramente propugnadas teóricamente, en algunos países, principalmente en los Estados Unidos (para la silosofia. de las reformas americanas, GoRE, 1993) y el Reino Unido (cf. METCALFE y RiCHARDS, 1989 (1987); ECHEBARRía, 1993), aunque según los contextos se haga más o menos énfasis en los diferentes objetivos particulares.

VILloRia, 1996, utilizando como criterios definitorios la mayor o menor preponderancia de contenidos tecnológicos o ideológicos en los programas de reforma, y el origen de las propuestas de reforma, según respondan a estrategias propiamente gubernamentales (asumidas por el Gobiemo en su conjunto o impulsadas desde la cabeza del mismo) o burocrático-corporativas (aunque estas últimas puedan inspirar propuestas de ministerios particulares), distingue cuatro modelos distintos de reforma:

1) El de aquellos países, como el Reino Unido, en los que el proyecto reformador es claramente ideológico y está postulado por el Gobiemo y el partido que lo sustenta.

2) El de aquellos países, como Estados Unidos, en los que el proyecto reformador es preferentemente tecnocrático, orientado primariamente a mejorar la eficacia del sector público. El proyecto también está postulado por el Gobierno.

3) El de aquellos países donde existe una Administración fuertemente descentralizada y con políticas sociales muy ambiciosas, como Holanda o Suecia. Los proyectos de reforma son postulados por Ministerios o agencias públicas particulares, y no globalmente por el Gobierno.

4) El de aquellos países, como España, donde los proyectos de modernización no tienen contenido ideológico, pero tampoco se da una postulación gubernamental ni partidista de tales proyectos, sino que son resultado del trabajo de sucesivos equipos en particulares ministerios (con predominio, en el impulso y diseño, del personal situado en la :zona gris" de delimitación entre políticos y burócratas).

\section{Causas y objetivos de las reformas administrativas}

\section{Causas}

No resulta fácil aislar las causas que determinan el auge de las reformas administrativas en .Occidentem en los últimos años, dada la incidencia de factores de muy diversa índole. El sector público está aquejado de múltiples enfermedades" (Hogwood y Peters, 1985, CAIDEN, 1991b), algunas quizá congénitas, de malformación del diseño de las organizaciones, otras derivadas de una deficiente nutrición de recursos. Sin embargo, las más parecen obedecer, por el contrario, a problemas de insuficiente asimilación de excesos nutricionales, con riesgo de infartos y otras crisis agudas. Uno de estos infartos, que obligó a adoptar terapias de choque, se produjo con la crisis económica del 73 , que obligó a las Administraciones públicas a adoptar curas de adelgazamiento. Buena parte de las reformas administrativas contemporáneas tienen origen en esta crisis económica que alertó sobre los riesgos del exceso de peso del sector público.

Las burocracias públicas no gozan de muy buena prensa (Veblen, 1904; Mises, 1974 (1962); MerTon, 1992 (1968); Hummel, 1994), y no sólo por "estar gordas". Tienen fama de corruptas, despilfarradoras, torpes y tiránicas. Son insultadas popularmente, satirizadas en crueles chistes, despreciadas por su falta de agilidad en la gestión de los asuntos públicos y odiadas por su aparente insensibilidad a las necesidades de los ciudadanos (son relativamente pocas las voces que se han levantado en su favor, por ej., BLAU y MEYER, 1971; GOODSELL, 1994).

De aquí la continua postulación de alternativas organizativas y de gestión a la burocracia (DRUCKER, 1988; HECKSER, 1994); pero estas alternativas inducen a comparaciones poco justas de la aneficaz burocracia. con seductores ideales de organización, no sometidos a la prueba de la práctica. Así, las reformas administrativas que prometen acabar con todos los males que aquejan a las Administraciones, situándolas en la Arcadia feliz de las organizaciones postburocráticas tienen la doble virtualidad de ofrecer a la generalidad de los políticos un chivo expiatorio con el que redimir su ineficacia a la hora de gobernar y, más concretamente, para aquéllos recién llegados al poder la oportunidad de proponer objetivos simbólicos que aparentan romper con los males del pasado.

La globalización mundial de la economía es también un recurso muy socorrido para justificar las reformas administrativas (LUKE y CAIDEN, 1989). Se suele indicar, desde este punto de vista, que la Administración pública es un elemento esencial en la competitividad de una nación (PORTER, 1991: 763-847) y, por 
tanto, las reformas son indispensables para adecuarse a las necesidades de la competencia intemacional (DuNLEAVY, 1994). Es frecuente que se incida especialmente, muchas veces de forma meramente retórica, en el ahorro de recursos y en la capacitación del personal del sector público (Rodríguez-Arana y Bastos BOUBETA, 1996).

Las reformas administrativas pueden ser un resultado no deliberado de reajustes en las relaciones de poder entre los distintos actores del proceso político (MARCH y OLSEN, 1983)'. Las reformas serían consecuencia de una combinación más o menos accidental de propósitos y oportunidades de elección por actores individuales no coordinados, problemas y soluciones. Constituirian un buen ejemplo del garbage can model of organizational change, en la expresión de COHEN, MARCH y OLSEN, que describe un proceso de toma de decisiones en las organizaciones en el que las soluciones preceden a los problemas: primero tendríamos los recursos, soluciones y técnicas para abordarlos y luego buscariamos los problemas concretos a los que hacer su aplicación.

No se debe ignorar la posibilidad de motivaciones más frívolas en el inicio de los programas de reforma administrativa. Del mismo modo que el vestido, la comida y las costumbres, la gestión pública no se sustrae a la moda. Naturalmente este condicionamiento dista de ser espontáneo. Tanto en referencia al ámbito de la gestión pública como de la privada existen una serie de gumis, que producen (pero, con disposición aindustrialn) "nuevas ideas" para una mejor gestión. Ideas legitimadas con palabras muy cargadas positivamente, pero poco precisas, como eficiencia, eficacia, calidad, productividad, progreso, etc. (ABrahamson, 1996). Estos gumis, en general con su corte de asesores académicos à la page, venden ssus saberes* en un lucrativo mercado que se beneficia de la demanda de innovaciones en la gestión por parte de políticos buscadores de votos o de políticos y altos burócratas deseosos de acrecer su legitimación como recurso de promoción profesional. Esto explica la fugacidad de muchas de las propuestas que se hacen en este terreno, siempre expuestas a ser superadas por otras ‘más avanzadas" o novedosas (Harari, 1997, con referencia a la última moda). RugBY, 1993, una de las voces mas críticas de estos desarrollos, ha elaborado un esquema del ciclo vital de estas "teorías" innovadoras de la gestión: (1) Constatación de deficiencias La inadecuación de algún principio importante de la gestión a cambios del entorno genera insatisfacción respecto del statu quo; (2) Descubrimiento de la solución. Pensadores creativos desarrollan nuevas soluciones o reactualizan viejas soluciones para corregir las deficencias, (3) Euforia. Los primeros relatos de los éxitos de las nuevas técnicas son altamente publicitados, eliminando las barreras procedentes de la inercia. Se alimenta, así, el espejismo de que la nueva técnica rresolverá de una vez y para siempre el probleman; (4) Sobredifusión. La euforia con- duce a la aplicación de la nueva técnica en situaciones inadecuadas, lo que conduce a resultados desalentadores. Las dificultades inducen un mayor énfasis aplicativo, para superar radicalmente los problemas ${ }^{6} ;$ (5) Ridiculización. Los ejemplos de fracasos llegan a ser demasiado obvios para ignorarlos. Los participantes y la opinión en general evidencian cruel y públicamente los defectos de la técnica; (6) Abandono. Las organizaciones descartan la nueva técnica y se abre el proceso de búsqueda de otra más funcional y ‘moderna.

Como hemos visto, las causas de las reformas son múltiples, y múltiples también las perspectivas de su contemplación. Generalmente, en cada caso se podrá establecer una jerarquía de causas en la etiología de la reforma admlnistrativa, aunque es probable que todas las consideradas anteriormente intervengan en algún grado. Veamos ahora, a grandes rasgos, cuáles son los objetivos de las reformas.

\section{Objetivos de las reformas}

Los objetivos de las reformas pueden ser más o menos explícitos. Por supuesto los objetivos explicitados son más fáciles de conocer que los no declarados, aunque de ello no se desprende que sean necesariamente más importantes. SuBIRATS y Gomá, 1993, resumen los objetivos oficiales de los programas de reformas:

1) Mejorar la competencia y capacidades técnicas. Ante la cada vez mayor complejidad de los problemas y la gran movilidad de los entornos de las Administraciones públicas, una de las soluciones pasa por mejorar la competencia técnica de las Administraciones y su capacidad analítica y de anticipación.

2) Cumplir y fortalecer los valores constitucionales. Las Administraciones públicas hoy en día cumplen funciones no sólo de prestación de bienes y servicios, sino también de negociación entre intereses parciales contrapuestos. La reforma de la Administración busca, pues, mejorar sus capacidades políticas, para hacer frente a un conjunto de intereses cada vez más fragmentarios y para orientar los servicios por unos valores que sirvan para mejorar la vida social.

3) Gestionar la complejidad, la incertidumbre y el cambio. Los modelos organizativos burocráticos regidos por una lógica de funcionamiento interno sometida exclusivamente lal Derecho administrativo resultan claramente insuficieñtes para abordar el abanico de problemas que se presentan a las Administraciones en la actualidad. Esta no-coincidencia entre las funciones requeridas y el mo- 
delo organizativo genera notables tensiones en el funcionamiento de la Administración. La reforma trata de adecuar la realidad de la Administración a esta compleji$\mathrm{dad}$, intentando superar las rigideces procedimentales que dificultan una debida atención a los administrados.

4) Actuar con una ética de servicio al ciudadano. Las reformas, según este objetivo, tratan de cambiar el trato a los administrados. De una consideración fundada en las nociones de súbdito o administrado se trata de pasar a otra fundada ya en la noción de cliente, lo que enfatiza los elementos bilaterales de la relación, ya en la más clásica de ciudadano, dotado de derechos frente a la Administración pública. Con estas reformas se busca mejorar la calidad del servicio público, incorporando al funcionamiento del sector público no sólo al ciudadano considerado individualmente, sino también a asociaciones y otros grupos o colectivos. Esta ética de servicio no sólo busca la resolución de problemas sino una mayor transparencia y comprensibilidad de la actuación administrativa para facilitar esa participación ciudadana.

De un modo pormenorizado los objetivos principales de las reformas contemporáneas, en nuestra opinión, son: (1) la postulación de la introducción y potencialización del gerencialismo en el sector público (PourtT, 1993); (2) de modo derivado o asociado, el intento de disminuir, y si es posible, revertir el crecimiento del sector público; (3) el incremento de la productividad de la Administración pública (Holzer, 1992; BaÑón, HalaCHmí y BoucKAERT, 1996); (4) el énfasis en la consideración de la provisión pública de servicios como meramente subsidiaria del sector privado, la huida de las normas del Derecho administrativo hacia las normas, más flexibles, del Derecho privado (Richards, 1992) y, más en concreto, la des-regulación de la prestación de servicios públicos y su cesión al sector privado (ARENLLA, 1994); (5) la difusión del consumer paradigm, cuya traducción más simple, y no inexacta, es la de paradigma del consumidor, pero que recibe otras traducciones ${ }^{7}$ (RICHARDS, 1994 (1992); JENKINS y GRAY, 1994 (1993), y la subsiguiente postulación de la calidad de los servicios; (6) la participación de ciudadanos y empleados en la gestión (BASTOS BOUBETA, 1995) y la mejora del desempeño de los funcionarios públicos, a través de la aplicación de incentivos al rendimiento, salariales o extrasalariales; (7) el énfasis en el desarrollo de la automatización de la gestión pública, especialmente en lo que se refiere a la incorporación de los desarrollos de las tecnologías de la comunicación; (8) el desarrollo de una agenda más sensible a objetivos de cooperación intergubernamental y a los condicionamientos internacionales en la elaboración de políticas (HoOD, 1991).

Pero además de esa agenda explícita, pueden existir agendas ocultas en los programas de reforma administrativa. Algunos de estos objetivos ocultos pueden ser la supresión de servicios administrativos que no encajen con los propósitos de los dirigentes políticos o les resulten molestos. Esto es especialmente palpable en buena parte de las reformas que se califican como gerenciales, en el propósito de recortar el poder de los altos funcionarios de carrera en provecho de la clase política, incrementando el poder de patronazgo de ésta. Por ejemplo, las ideas que impulsaron las reformas británicas enfatizaron mucho en la descripción del burócrata como un individuo egoísta que no busca satisfacer el bien común, sino sólo el suyo propio (NISKANEN, 1971, es especialmente representativo de esta perspectiva, cf., ASHFORD, 1993) y que acumula a través de su maquiavélico control de la elaboración de los presupuestos y de su dominio de las interioridades del proceso de elaboración de políticas un desmesurado poder que suplanta el poder legítimo del político elegido por el pueblo ${ }^{8}$. Entre nosotros, ALBA (1995: 399), refiere la opinión de un Ministro, en la etapa de la transición, que describía a los funcionarios y sus organizaciones como peligrosos toros de lidia.

Los burócratas podrían así, constituir un gran obstáculo a la hora de implementar políticas. El relato de los padecimientos de Rudolf Hilferding, quien en 1923 vio fracasar su políica económica ante la resistencia de la vieja burocracia prusiana, que veía lesionados sus intereses de "casta", ha sido aducido en la bibliografía para advertir contra los males del excesivo poder funcionarial (PaGe, 1992: 1). En este ambiente, sesgadamente predispuesto contra el burócrata, es lógico que las reformas administrativas busquen recortar el peso político de la burocracia y aumentar el de los políticos, particularmente como empleadores. (reclutamiento, promoción y sanción del personal al servicio de las Administraciones públicas). En este aspecto, las reformas administrativas pueden ser vistas, en bastantes casos, como una lucha latente entre políticos y burócratas (PETERS, 1992) con el objetivo no declarado de reducir la participación de los segundos en la elaboración e implementacion de políticas.

\section{Las reformas administrativas en España}

Las reformas administrativas en la Administración central

La Administración pública española -como la de la generalidad de los países - ha cambiado notablemente (sobre sus antecedentes modernos, GARCía DE ENTERRIA, 1961; sobre las reformas, SuBIRATS, 1990). Los grandes cuerpos de burócratas han perdido influencia respecto de los años setenta y los niveles superiores de la función pública han bajado en capacitación profesional y prestigio social. La Administración central del Estado ha sufrido 
un cierto proceso de adelgazamiento por la institucionalización de las autonomías territoriales, pero este proceso ha sido contrarestado por el crecimiento de los servicios públicos de educación y seguridad social, no transferidos a todas las Comunidades Autónomas, o no en su totalidad, en los casos en que sí se ha producido la transferencia.

A partir de mediados de los años ochenta, más en concreto tras la aprobación de la Ley de reforma de la Función Pública de 1984 y la acentuación del proceso de transferencias a las $\mathrm{Co}$ munidades Autónomas, potenciados quizá por factores externos ${ }^{9}$, se inician una serie de reformas de la Administración central del Estado. Estas reformas han sido consideradas por la generalidad de los analistas en términos muy críticos (ALBA, 1995). Sus principales características, según NiETO, 1996 (cf., CANALES, 1993), fueron:

En primer lugar, se hicieron públicos una serie de documentos oficiales sobre las reformas, como las Reflexiones para la modernización de la Administración, del MAP; el Estudio Delphi: la modernización de los procedimientos de actuación de la Administración, de la Inspección General de Servicios; el Plan de Modernización de la Administración del Estado (aprobado por el Consejo de Ministros en noviembre de 1991 y marzo de 1992); y el documento-marco sobre Modernización y cambio en las Administraciones Públicas, del INAP, 1995. Paralelamente se celebraron varias jornadas de estudio, auspiciadas institucionalmente, sobre modernización y reforma administrativa, como las "Jornadas de modemización de las Administraciones Públicas. (Cuenca, julio de 1990), "Modernización pública y formación" (octubre de 1991) o el "Seminario Internacional sobre la Reforma Administrativav (Santiago de Compostela, 1995).

Los contenidos varían según los casos, pero las notas comunes incluyen propuestas de reconfiguración de las estructuras administrativas (creando agencias. ${ }^{10}$ y entes autónomos), de transformación de *culturas burocráticas" en culturas de responsabilidadn ", de flexibilización de los sistemas de función pública (BAENA DEL ALCÁzAR, 1996) y de la mejora de la información y comunicación con el ciudadano y de la calidad de los servicios (creación de un Observatorio de la calidad) ${ }^{12}$.

La reforma administrativa tiene también un componente de reforma de la legislación, en un intento de superar el control endógeno de la Administración por parte de los altos cuerpos de funcionarios (nos remitimos a lo dicho sobre los propósitos de incremento del poder de patronazgo por los políticos), mediante la introducción de la movilidad en el puesto de trabajo y de la "carrera administrativa", incluso entre distintas Administraciones Públicas, la racionalización y recomposición de los Cuerpos de funcionarios y la limitación de los puestos de libre designación (ORTEGA, 1996: 19), objetivo este último, contradictorio con el espíritu de la re- forma, pero, quiza por ello mismo, incumplido en la práctica. Esta actuación se ha concretado en normas como la Ley de Incompatibilidades y la Ley de Régimen Jurídico de las Administraciones Públicas.

Las reformas administrativas realmente implantadas, con algunas excepciones como la creación de la Agencia Tributaria o la reforma de la Dirección General de Tráfico (cf., VnLoRIA, 1996), no pasan de ser, usando la expresión del propio Alejandro NIETo, intervenciones epidérmicas y meramente miméticas del ‘discurso modernizador. imperante allende nuestras fronteras ${ }^{13}$. Pero, los resultados prácticos han sido muy modestos, y las relaciones de poder entre políticos y burócratas permanecen sustancialmente inalteradas (dentro de la mutua desconfianza que, según Beltrán Villalva, 1996, las caracteriza). Impera un discurso retórico plagado de lugares comunes, como tales perfectamente compatibles aun siendo contradictorios, como da Administración ni se cambia por decreto ni se transforma de la noche a la mañana. 0 da modernización tiende a un cambio de cultura administrativa centrado en la consecución de resultados y en el equilibrio de la relación coste beneficio como elemento referencial básicon (cf., NIETO, 1992), entregado a las sucesivas y fugaces modas. Si en los años ochenta se enfatizaban las nociones de eficiencia y eficacia ${ }^{14}$, en los noventa se insistirá en el paradigma del consumidor (o cliente) RICHARDS, 1994 (1992), más conciliable con el nuevo ambiente "humanista. (AtKouf, 1992), de mayor predicamento en la actualidad.

En conclusión, aunque la Administración española sufrió, en los últimos veinte años, el impacto de una reforma legislativa de cierta entidad, como la Ley de la Función Pública de 1984, y algunos otros cambios menores, a los que se ha aludido en las páginas anteriores, el cambio más importante ha venido constituido por la aparición de las 17 Administraciones autonómicas. Veremos a continuación las reformas acometidas en las Comunidades Autónomas.

\section{Las reformas administrativas en las Comunidades Autónomas}

Las Administraciones autonómicas hispanas comienzan su andadura en los años ochenta, en un contexto de pleno apogeo de las reformas administrativas en toda Europa. Sin embargo, no se aprovechó la ocasión. para introducir tipos nuevos de Administracion pública, de acuerdo con los postulados de los profetas de la reforma administrativa, lo que probablemente es debido más a las dificultades reales que encuentra la implemen- 
tación de estas propuestas arbitristas que a la pereza mental de los respectivos responsables políticos.

Por el contrario, el modelo seguido a la hora de crear estas nuevas Administraciones fue el de la vieja y tradicional. (y probablemente más eficaz de lo que se quiere reconocer) Administración burocrática, copiada con sus virtudes y defectos de la Administración central del Estado. Por ello, la Administración de cada una de las Comunidades Autónomas es semejante, a la vez, a la Administración central y, en principio, también a sus hermanas autonómicas. RAmió y SuBIRATS (1996) afirman lo primero, pero relativizan mucho lo segundo: según ellos, por debajo de un dibujo aparentemente homogéneo aparece una plenitud de matices, diferencias, desigualdades y coloridos distintivos. Y estos contrastes mostrarían estrategias o formas de entender y usar la autonomía diferenciadas. Desde luego, es verdad que las formas de comprensión de la autonomía y las estrategias de su utilización varían en gran medida, pero nosotros creemos que estas diferencias no se han proyectado en las reformas administrativas, que concuerdan básicamente en sus contenidos.

Por otro lado, las propuestas de reformas administrativas pueden ser una estrategia de diversión ante problemas políticos más graves o perentorios. Al respecto, CASTELLS, 1992, cuenta que André Tardieu, Presidente del Consejo de Ministros francés en el período de entreguerras, cuando aumentaba la tensión política y el Gobierno se encontraba en dificultades anunciaba en la Asamblea Nacional una reforma administrativa, anuncio que era recibido con regocijo derivado de la incredibilidad que suscitaba, lo que permitía al Primer ministro lograr la relajación de la tensión parlamentaria suscitada por aquellos otros problemas. Los políticos autonómicos pronto percibieron estas potencialidades de las propuestas de reformas administrativas. De ahí que las reformas autonómicas hayan sido en general bastante superficiales y que, al mismo tiempo, la difusión por mimesis de sus contenidos haya sido muy elevada. En relación a los Estados Unidos, InGRAHAM, 1993, señala cómo las políticas (en el caso concreto, de remuneración de funcionarios) que parecen funcionar bien en una particular Administración regional se difunden rápidamente en todas las demás Administraciones regionales, sin estudiar si las particulares circunstancias de cada una permiten esperar resultados análogos.

Una vez institucionalizadas y alcanzado un nivel suficiente de competencias, a comienzos de los años 90 las Comunidades Autónomas, en ocasión muchas veces de cambios en los gobiernos, se embarcan en procesos de reforma admmistrativa. Un simple repaso de la bibliografía reciente (monográficos de Autonomies, n. ${ }^{\circ} 18$, y Revista Vasca de Administración Pública, $\mathrm{n}^{\circ}$ 45-II, y las comunicaciones presentadas a diversas Jomadas sobre Modernización y Reforma en las Comunidades Autónomas) nos muestran muchos rasgos comunes entre ellas:
La mayoría de los programas de acción son prograrnas modernizadores, no propiamente de reforma. Buscan adecuar, eso sí, con una retórica de eficacia y aproximación al ciudadano (Cf., AZÚA, 1991; FLORENSA, 1991; VILORIA, 1994), los avances técnicos derivados de la informatización a una mejor atención al ciudadano ${ }^{15}$, creando sistemas informáticos que permitan el acercamiento de los ciudadanos a las gestiones administrativas. En cambio, no se procura de forma deliberada un cambio en la ccultura. administrativa ni en el sistema de gestión de recursos humanos.

Los pocos casos de verdaderas reformas se circunscriben a procesos, aún tímidos, de evaluación del rendimiento y de medición de la productividad de los empleados públicos. Hasta ahora, en los casos en que se han implantando evaluaciones del rendimiento no tienen consecuencias sobre la retribución del personal. Sus objetivos son, como dicen LOSADA y XIRAu, 1995, para el caso catalán, aumentar el grado de identificación de los trabajadores con el servicio público, reforzar el ejercicio de las funciones directivas por parte de los mandos, obtener informaciones aplicables a los procesos de provisión de puestos de trabajo y de planificación de actuaciones formativas y, por último, introducir elementos que favorezcan la implantación de sistemas de dirección por objetivos.

Muestran, por último, si cabe aún más, que en la Administración central del Estado, excesos retóricos, con reiteración abusiva de palabras tales como eficacia, atención al ciudadano y acercamiento de la Administración al ciudadano.

\section{La reforma administrativa en la Comunidad Autónoma de} Galicia

El deseo de contar con una Administración pública propia de Galicia fue uno de los ideales que siempre animaron y siguen animando el pensamiento regionalista y nacionalista gallego (González Mariñas, 1993; Vilas Nogueira, 1993; YeBra, 1993).

La Administración autonómica gallega ha seguido un proceso sostenido de crecimiento en competencias y en volumen de presupuesto, que ha llegado a alcanzar el 15\% del PIB de la Comunidad (GAGO y ÁlVAREZ VILLAMARín, 1992). Igualmente, ha registrado un aumento importante en el volumen de personal, que ha llegado a significar casi el $9 \%$ del total de la fuerza de trabajo de la Comunidad, lo que aun siendo relativamente bajo en relación a otras Comunidades Autónomas (GARCÍA LOPEZ y ALVIRA, 1996), representa un porcentaje considerable (sobre la función pública gallega, cf., LÓPEZ-RúA, 1991). 
A la hora de crear esta Administración se siguió, del mismo modo que en las restantes Comunidades Autónomas, miméticamente el modelo de la Administración central del Estado, tanto de las estructuras generales territoriales como de los organismos autónomos. Entre los segundos, los hay de muy diferente tamaño y naturaleza jurídica, desde el Servicio Galego de Saúde, que consume más de la mitad del presupuesto de la Comunidad Autónoma a otros más modestos como el Instituto Galego da Vivenda e Solo, la Televisión de Galicia o la Escola Galega de Administración Pública (Rodríguez-Arana, 1993; García Llovet, 1994; Rodríguez-Arana y Aguilar, 1994).

Los movimientos de reforma administrativa en Galicia comienzan en el año 1991, coincidiendo con el fin de la etapa de rápida expansión de las competencias autonómicas y con el regreso al Gobierno de la Comunidad del Partido Popular, como fruto del impulso del conselleiro de la Presidencia, Dositeo RoDRíGuEz ${ }^{16}$. La continuidad de este conselleiro ha permitido que la reforma goce de impulso sostenido, aspecto muy importante para su éxito. Veamos ahora en qué consiste:

Administración Unica. Es una propuesta de carácter político-administrativo, referida inicial y principalmente a las relaciones entre la Administración del Estado y las Administraciones autonómicas, lanzada desde la misma Presidencia de la Xunta de Galicia (FraGa Iribarne, 1993; cf., Serna, 1993). En base al principio de subsidiariedad se postula que la prestación del servicio público se realice por la Administración territorial más próxima al ciudadano, siempre que tenga capacidad para hacerlo según la naturaleza del servicio. En opinión de Meilán (1993) no es otra cosa que el reajuste, pendiente, de la Administración central para su adecuación al Estado autonómico, consistiendo en la práctica en la asunción por la Comunidad Autónoma de las competencias de la Administración periférica del Estado (cf., RoDriguEzARANA, 1997).

Comarcalización. La Xunta de Galicia está inmersa en un proceso, reclamado históricamente desde el galleguismo (cf., PreCEDO, 1993) y que tiene su fundamento legal en el reconocimiento por el Estatuto de Autonomía de la comarca como uentidad local propia de Galicia* (sic) (cf., FerReIRA, 1991), de reorganización administrativa y dotación de competencias a las comarcas. A este propósito se han creado unas Fundaciones para el desarrollo comarcal, todavía en fase incipiente.

Codificación nommativa. Entre las propuestas de modernización administrativa ha tenido especial aceptación en Europa continental, sobre todo en Francia (MatTarella, 1993; BralBANT, 1994), la codificación de las normas jurídico-administrativas para eliminar duplicidades, permitir su simplificación y facilitar su tratamiento informático. Se trata de sintetizar y cuando sea preciso refundir las normas administrativas (SER-
NA, 1994) para un mejor funcionamiento de la Administración, en particular una mayor racionalización del procedimiento administrativo, simplificación de trámites y una mejor garantía de los derechos ciudadanos en el procedimiento (Alvarelos, 1993).

Reforma de la función priblica. La mejor gestión de los recursos humanos empleados en la Administración autonómica, la mejora de su estructura administrativa, atacar la rutina y el burocratismo, y despolitizar la Administración son los principales objetivos buscados con la reforma de la Ley de la Función Pública gallega (Rodríguez Rodríguez, 1994). En particular, se busca flexibilizar las plantillas de personal, llegando incluso a la movilidad espacial, facilitar la planificación de recursos humanos a través de la previsión de necesidades de efectivos disponibles y necesarios, cierta flexibilización de los procedimientos de selección de personal y provisión de puestos de trabajo y la exigencia de un diploma de directivo para el acceso a los puestos de libre designación (BAENA DEl ALCÁzAR, 1994).

El sistema de gestión de procedimientos administrativos (SGPA). Es una aplicación de herramientas infórmáticas ${ }^{17}$ para acercar la Administración al ciudadano y simplificar los trámites administrativos (cf., MárQUEZ, 1995). Trata de incrementar la eficacia, racionalidad y homogeneidad de la gestión pública en la Administración autonómica, mediante el seguimiento de los procedimientos administrativos. Abarca todas las Consellerías de la Xunta y sus delegaciones provinciales (BOUZADA, 1994b), incluyendo todos los procedimientos que se inician a instancia de parte ${ }^{18}$.

En particular, persigue dos objetivos:

a) Acercamiento al administrado mediante la creación de órganos específicos de carácter comarcal con funciones de admisión de documentación y de información general, y mediante el suministro de información acerca de la actuación de la Administración por medio de un servicio de atención telefónica y de edición de guías de atención al ciudadano.

b) Simplificación del proceso de gestión administrativa, mediante la instauración de un sistema de procedimientos que acerque a la Administración autonómica a los postulados de la calidad total (VAZZuEz ARES, 1994). Esta simplificación requiere una cualificación específica del personal y la identificación y racionalización de los procedimientos administrativos iniciados a instancia de parte. Los principales órganos encargados de las diferentes funciones del proceso son (cf., MárQuEZ, 1995):

- Órganos de coordinación: Comisión Central de Racionalización. Administrativa y Comisiones de Racionalización propias de cada Consellería. 
- Órganos técnicos: Comisión Central de Revisión de la Normativa y Comisiones de Revisión propias de cada Consellería.

- Órganos de control: Inspección General de Servicios.

Evaluación del rendimiento. Persigue efectuar una valoración anual de todas las unidades administrativas, con nivel de subdirección general y jefatura de servicio, a fin de estimar su rendimiento (BouzADA, 1995). El objetivo concreto es valorar la cantidad y calidad del trabajo realizado en referencia a:

a) la organización de la unidad y el resultado obtenido en relación con los medios personales y materiales puestos a su disposición; b) la capacidad organizativa propuestas y mejoras introducidas en la organización y funcionamiento de la unidad a instancias de su titular.

A estos fines se ha creado una Comisión de Evaluación del desempeño, que emitirá su evaluación previo informe de la Inspección General de Servicios. Se prevé la posibilidad de aplicar los resultados de la evaluación a la valoración de los puestos de trabajo y a la determinación del complemento de productividad, pero a corto plazo no afectará a la remuneración de los empleados (BOUZADA, 1994a).

Respecto a las reformas administrativas emprendidas por la Comunidad Autónoma de Galicia cabe hacer varias precisiones:

En primer lugar, es conveniente distinguir la reforma de las actuaciones modernizadoras, o de adaptación de la Administración a las alteraciones de su entorno, tecnológico, económico o social. La codificación normativa y el Sistema de Gestión de Procedimientos Administrativos se limitan a aplicar nuevas técnicas, particularmente de tipo informático. Constituyen una mera adaptación a la dinámica tecnológica y social circundante, eso sí, impregnada de una nueva afilosofía. de servicio al consumidor o cliente y expresiva de una notable celeridad de respuesta a los cambios de métodos.

Consideración particular también merecen, en segundo lugar, las reorganizaciones territoriales del poder político. La reforma de la estructuración territorial de la Administración ha de diferenciarse de aquellas otras centradas en los estándares de gestión (cuestiones de calidad, productividad, gestión de recursos humanos, etc.) o de la relación entre la Administración y los destinatarios de sus productos (paradigma del administrado, del consumidor, etc.), que son las que suelen ocupar la bibliografía contemporánea sobre la reforma, aunque las reformas territoriales puedan incidir secundariamente en estos aspectos: acercamiento de las instancias administrativas al ciudadano, en el caso de la comarcalización; evitar duplicaciones innecesarias en la prestación de servicios, en el caso de la Administración Única.

Las actuaciones que, a nuestro juicio, merecen verdaderamente el calificativo de reformas administrativas, en el sentido antes precisado, son la modificación de la Ley de la Función Pública y la Evaluación del Rendimiento. La Ley de la Función Pública gallega trata de introducir una mayor flexibilidad en la gestión de recursos humanos. Pudiera parecer poco ambiciosa en comparación con otras reformas europeas, inspiradas en la homologación de los criterios de contratación del personal al servicio de la Administración con los del sector privado, pero a este propósito han de tenerse en cuenta no sólo elementos muy arraigados de nuestra cultura política, sino incluso ciertas constricciones constitucionales. Posiblemente, el problema se plantee en sentido contraric: la cautelosa flexibilización emprendida se contradice con el propósito declarado de reducir la politización de la Administración, pues a mayor discrecionalidad en la contratación y promoción de los empleados, más poder para el órgano político y menos para el burócrata.

La reforma que nos parece más interesante es el prograrna de Evaluación del Rendimiento. Constituye un intento muy importante (aunque, de momento, no tenga consecuencias retributivas para los funcionarios evaluados), en la línea de atras Administraciones occidentales. Uno de los principales problemas que se plantean a este tipo de reformas es el de la determinación de los indicadores del rendimiento. Es una cuestión muy compleja, a la que no hay respuestas claras: ¿Qué se ha de entender por productividad? ¿Cómo comparar la productividad de dos unidades completamente distintas? ¿Cómo medir la calidad de la atención? Otro problema importante es el de las resistencias que suelen manifestarse a la implantación de estas reformas. A nadie le gusta que se le mida y compare su trabajo y más en lugares como la Administración Pública, donde no hay tradición de hacer tal cosa.

En conclusión, la reforma administrativa de la Comunidad Autónoma de Galicia merece un juicio positivo; sale bastante bien parada en la comparación con las emprendidas por otras Administraciones. Los aspectos más positivos son la determinación política para la reforma, la sensibilidad hacia los imperativos de cambio y su rápida respuesta y, sobre todo, el programa de evaluación del rendimiento, innovador en nuestro entorno ${ }^{19}$. Aspectos menos positivos son los excesos retóricos en la presentación de las reformas, un sentimiento de inferioridad respecto al sector privado y un seguimiento inconsciente de las modas de gestión, que pululan por el sector público, extremos éstos en que la reforma gallega se pliega a inspiraciones generalizadas en España. 
- Catedrático de Ciencias Politicas y de la Administración. Universidad de Saniago de Compostela.

- Profesor Ayudante de Ciencias Políticas y de ba Administración. Universidad de Santiago de Compostela.

' Un ejemplo significativo: Eduardo ZAPICO y LE. ECHEBARRí tadujeron el Improving Public Management, de METCAufe y Richards (1987) por La modermización... No es la mejor opción desde el punto de vista estrictamente léxico, pero se corresponde con la concepción de (vide nota 3).

' Brabavt, 1992: 57 , define la modemización administrativa como una puesta al día permanente de la Administración, en función de las exigencias del momento y del lugar.

"ECHEBARRIA (ETXEBARRIA,1989, y ECHEBARRIA, 1993: 140-145) ha distinguido entre cambios planificados $e$ inercias administrativas, $o$ actuaciones meramente reactivas, pero para él la modemización es, precisamente, el cambio planificado.

1 Sin embargo, de hecho, como atestiguan numerosos estudios empiricos, la paricipación de los burócratas en la elaboración de politicas es muy relevante, Cf., por ej., ABERBaCH, PUTNam y Rockmav, 1981; para España, cf. OlmEDa, 1988.

' En cambio, para algunos estudiosos de las políticas públicas, las políticas de reforma administrativa son consideradas en el grupo de las políticas institucionales, como políicas que buscan la producción, transformación o mejora de instituciones públicas. En general son poco onerosas, pero en cambio conllevan importantes alteraciones en las relaciones de poder (QUERMONNE, 1985).

' ${ }^{6}$ No es necesario ser devoto de Freud para constatar que, con frecuencia, las resistencias del entomo a los propósitos de la acción actúan como estímulos adicionales para persistir en estos propósitos. En el caso, no infrecuente, de que aquellas resistencias se deban a enores estratégicos, se acomete, asi, un camino de perdición.

'Veamos un ejemplo muy significativo: en el n." 1 de Gestión y Análisis de Politicas Puiblicas, el articulo de S. RICHards (1992), Who Defines the Public Good? The Consumer Paradigm...., es traducido por J. Subirats como .El paradigma del cliente...; en cambio, el arículo de B. Jenkins y A. GraY, •Evaluating and the Consumer... (1993) es traducido por $\mathrm{X}$. Ballan como .Evaluación y el usuario.... Las implicaciones de una u otra opción son enteramente distintas.

En español, la palabra * cliente designa al comprador de productos suministrados por empresas industriales y mercantiles (dejando al margen, obviamente, otras significaciones más próximas a la originaria romana, que no vienen al caso aqui). En cambio la palabra usuario designa a la persona que utiliza un servicio público, en el sentido del Derecho administrativo o en un sentido más general, servicio susceptible de uso por el -público, utilización que no necesariamente supone el pago de un precio (por ej., los usuarios de las vias públicas; no de peaje). La traducción más literal -consumidor. evitaria estos problemas, sin que se pueda decir que alterase el significado original.

${ }^{8}$ Una visión satírica del entomo de Margaret Thatcher, en esta perspectiva de los políticos como peleles en manos de astutos burócratas que controlan la verdadera elaboración de las politicas, en Jonathan LYsv y Anthony JaY, Si, Ministro (trad. espanola), Barcelona, Ultramar, 1985.

9 LASHERAS y RUIZ-HUERTA, 1992, sostienen que el proceso de unificación europeo, con sus exigencias de racionalización del gasto público, jugó un papel espoleador de las reformas.

1n La extemalización. de las acividades administrativas en agencias. es, en general, una de las caracteristicas de las reformas de los noventa (JENKINS y GRAY, 1994 (1993): 47).

"Sobre este tópico, cf., Jenkivs y Gray, 1994 (1993). No es dificil constatar, en los documentos oficiales sobre las reformas y en la mayor parte de la bibliografia sobre la cuestión, una trivialización del término ccultura., que suponemos homorizaria a Almond y demás inventores de la cultura política.

12 Una sintesis auténtica del discurso oficial sobre la reforma administrativa en la comunicación del, a la sazón, Ministro para las Administraciones Territoriales, Jerónimo Saavedra, Reforma y modemización de las Administraciones públicas, presenta. da al Seminario Intemacional sobre la Reforma Administrativa, Santiago de Compostela, 1995 (mimeo).

13 Por ejemplo, el discurso del consumidor o cliente y de la calidad de servicio. se difunde en la retórica española sobre la Administración pública a partir de la publicación del informe de la OCDE, La Administración al senvicio del priblico, Madrid, MAP, 1991. Se populariza con la iraducción de Reinuenting Govemment (OSBORNE y GAE. BLER, 1994 (1992)).

${ }^{14}$ Se hablaba de las tres es. (economia, eficiencia y eficacia) e, incluso de las rinco es (añadiendo, excelencia y empresa). Cf., Elcock, 1989: 1-3.

" $\mathrm{Cf}$., las comunicaciones presentadas por los respectivos Consejeros de Andalucia, Aragón y La Rioja en las Jomadas sobre modemización y reforma administrativa en las Comunidades Autónomas, Santiago de Compostela, 27-28 de febrero de 1997 (en mimeo).

is Véase el primer proyecto de reforma en Rodriguez RODRIGUEZ, 1991, donde se insiste en los tópicos característicos de las reformas administrativas, como la racionalización de procedimientos y el acercamiento al ciudadano.

${ }^{17}$ Cf., Xunta de Galicia, El papel de los sistemas de información en el programa de reforma administrativa, Santiago de Compostela, 1997.

${ }^{18}$ Cf., Xunta de Galicia, A Reforma Administrativa da Administración Autonómica de Galicia, Santiago de Compostela, Xunta de Galicia, 1995.

${ }^{19}$ Las reformas administrativas gallegas son en general expresivas del •modelo hispánico de reforma administrativa: reformas no ideológicas e iniciadas en el seno de la Consellería de Presidencia; en cambio, la evaluación del desempeno proviene de una cultura administrativa más orientada a la productividad, como la nonteamericana, radicando en ello buena parte del atractivo que presenta su puesta en marcha. Puede servir de prueba para la adaptación de otros programas de reforma procedentes de aquel ámbito.

\section{Bibliografia}

Aberbach (Joel), Putnam (Robert D.) y Rockman (Bert A.) (1981), Bureaucrats and Politicians in Westem Democracies. Cambridge (Mass.)? Harvard Univers. Press.

ABRaHamson (Eric) (1996), :Management Fashion; Academy of Management Rev., vol. 21, n." 1, pp. 254-285.

ALBA (Cartos R.) (1995), L'Administration Publique Espagnole: Reforme ou Modemisation?. Rev. Francaise d'Administration Publique, n. 75, julio-septiembre, pp. 387-399.

Alvareluos (Constantino) (1993) et al., Reforma administrativa e procedimento administrativo, Santiago de Compostela, Xunta de Galicia.
ARENiLn (Manuel) (1994), Crisis de los recursos públicos, gestión pública y tendencias privatizadoras, en Manuel Arenilla, ed., Gasto Priblico y Crisis Económica, Santiago de Compostela, EGAP, 1994 (de hecho, 1995), pp. 25-54.

ASHFORD (Nigel) (1993), .The ideas of the new right, en Grant Jordan y Nigel Ashford, eds., Public policy and the impact of the new right, London, Pinter Publ.

ATKouf (Omar) (1992), -Management and the theories of organization in the 1990's: Toward a critical radical humanism., Academy of Management Rev., vol. 17, n. ${ }^{\circ} 3$, pp. 407-31. 
AzÚ (Jon Imanol) (1991), ·La calidad en la Administración Pública, Bol de Estudios Económicos, vol. 46, n. 143, agosto, pp. 309-318.

BaEva del Alcizar (Mariano) (1994), •A Administración de personal no Proxecto de Lei de reforma da Función Pública de Galicia, en Mariano Baena del Alcazar, ed., $A$ reforma da Función Priblica Galega. Santiago, EGAP, pp. 33-48.

BAENa del AlCizar (Mariano) (1996), .la modemización administrativaי, Revista Vasca de Administración Priblica. n. ${ }^{\circ}$ 45-II, mayo-agosto, pp. 13-15.

BANON (Rafael), HALACHMI (Arie) y BOUCKAERT (Geert) (1996), eds., La productividad y la calidad en la gestión pública. Santiago de Compostela, Escola Galega de Administración Pública.

Bastos Boubeta (Miguel A.) (1995), :Unha apertura plumalista da Administración, Rev. Galega de Administración Priblica, n. ${ }^{\circ} 11$, PR 171-187 (Santiago de Compostela).

Beitrái Vilialva (Miguel) (1996), ·De la reforma de la Administración al control de calidad de los servicios públicos, Gestión y Análisis de Politicas Priblicas, n. ${ }^{\infty}$ 5-6, enero-agosto, pp. 5-16.

Blau (Peter M.) y MeYer (Marshall W.) (1971), Bureaucracy in Modern Saciety, 2.' edic., New York Random House.

Bouzada (Jaime) (1994a), •A avaliación do rendemento no Proxecto de Lei da Función Pública de Galicia, en Mariano Baena del Alcázar, ed., A reforma da Función Pública Galega, Santiago, EGAP, ..., pp. 23-32.

Bouzada (Jaime) (1994b), •A reforma administrativa autonómica de Galicia, en Xaime Rodriguez-Arana, ed., Reforma administrativa. Seminario bilateral bispano-alemän sobre reforma administrativa. Santiago de Compostela, EGAP, pp. 89-98.

Bouzada (Jaime) (1995), -El papel de la Inspección General de Servicios en la Reforma Administrativa de la Xunta de Galicia: especial consideración de la evaluación del rendimiento, Gestion y Análisis de Politicas Priblicas, n. ${ }^{\circ}$ 2, enero-abril, pp. 79-84.

Braibant (Guy) (1992), .Qu'est-ce que la modemisation., Rev Franfaise d'Administration Publique. n." 61, enero-marzo.

BralBant (Guy) (1994), •A codificacion francesa (trad. al gallego), en Constantino Alvarellos, Francisco J. Sema y Guy Braibant, Codificacion normativa, Santiago de Compostela, EGAP, pp. 63-80.

CAIDEN (Gerald E.) (1991a), Administrative Reform Comes of Age, Berlin/New York Walter de Gruyter.

CAIDEN (Gerald E.) (1991b), What is really maladministration, Public Administration Rev., vol. 51, n. 6 , noviembre-diciembre.

CANalES (José Manuel) (1993), Notas sobre la modemización administrativa, en Izquierda Unida, Administraciones y Servicios Püblicos. ¿Reforma o privatización? Madrid, pp. 181-187.

CASTEus (José Manuel) (1992), • Notas póstumas sobre la reforma de la Administración pública española•, Rev. Vasca de Administración Priblica, n. ${ }^{\circ}$ 34 II, pp. 27-32.

DrUCKER (Peter E.) (1988), The Coming of the New Organization, Harvard Business Rev., vol. 88, n. ${ }^{n} 1$, enero-febrero, pp. 45-53.

Dunlfavy (Patrick) (1994), .The globalization of public services production: Can government be best in world?., Public Policy and Administration, vol. $9, \mathrm{n}{ }^{\circ} 2$, verano, pp. 3664 .

ECHEBARRIA (Koldo) (1993), La Administración priblica en la era del management (1esis doctoral), Univ. de Deusto (mimeo).

ELcock (Howard) (1989), . The three E's: Necessary but not sufficient conditions for good government, Public Policy and Administration, vol. 4, n. ${ }^{\circ} 1$, primavera.

ETXEBARRIA (Luis E.) (1989), -El cambio y la gestión del cambio en la Administración Pública (cambio planificado vs. inercia administrativa), en Instituto Vasco de Administración Publica, Modemización Administrativa, Oñati, pp. 21-48.

FerRejRa (Antonio J.) (1991), •Aproximación ó réxime xurídico da comarca•, en Xaime Rodriguez-Arana, ed., Estudios sobre o Estatuto galego, Santiago de Compostela, EGAP, pp. 447-460.

FLORENSA (Marisa) (1991), -La reforma administrativa en la Comunidad Autónoma de Cataluña. Rev. Galega de Administración Priblica, n. ${ }^{\circ}$ 2, pp. 121-150.

FraGa IRIBARve (Manuel) (1993), •Administración única: unha proposta para un verdadeiro Estado das Autonomias, en Xaime Rodríguez-Arana y Francisco J. Serna, eds., Administracion Unica. Relatorios das Xomadas celebradas en setembro de 1992 na EGAP, Santiago, EGAP, pp. 13-26.
GaGo (Alberto) y Álvarez Viluamarin (José C.) (1992), •El sector público autonómico en Galicia: La experiencia de una década (1983-19923, Presupuesto y Gasto Rúblico, ก. 7, pp. 103-125.

GARCI DE ENTERRIA (Eduardo) (1\%1), La Administración española, Madrid, Instituto de Estudios Políticos.

Garcí Llovet (Enrique) (1994), .la Administración institucional de la Comunidad Autónoma de Galicia. Algunas reflexiones, en Alfonso Pérez Moreno, ed., Administración instrumental. Libro bomenaie a Manuel Francisco Clavero Arévalo, Madrid, Instit. Garcia Oviedo. Univ. Sevilla/Ed. Civitas, pp. 1601-1620.

GarCh López (José) y Alvira (Francisco) (1996), •Personal al servicio de las Administraciones públicas, Papeles de Economia Española, n. ${ }^{\circ}$ 68-I, pp. 199-206.

GOODSEL (Charles T.) (1994), The case for bureaucracy. A public Administration polemic, 3.' edic., Chatham (New Jers.) Chatham House.

GONzNizz MARINas (Pablo) (1993), •A Administración galega na súa perspectiva histórica: cuestiones e problemas de organización..., en Xosé R. BARREIRO FERVANDEZ y Pablo GONZALEZ MARINAs, eds., Historia da Administración Puiblica. Relatorios $e$ comunicacións presentados... Santiago, EGAP, pp. 13-38.

GORE (A1) (1993), Crear una Administración puiblica que funcione mejor y cueste menos (trad. española), Instituto Vasco de Administración Pública.

Harar (Oren) (1997), •PPor qué murió la reingenieria?• (trad. de .Why Died Reenginering Die?., Management Review), Harvard Deusto Business Review, n. 77 , marzo-abril, pp. $26-30$.

HeCKSCHER (Charles) (1994), .Defining the post-bureaucratic type, en Charles Heckscher y A. Donnellon, eds., The post-bureaucratic organization. New York, Sage, pp. $14-62$.

Hocwood (Brian W.) y Peters (B. Guy) (1985), The pathology of public policy, Oxford, Clarendon Press.

Holzer (Marc) (1992), ed., Public Productivity Handbook. New York, Marcel Dekker.

HoOD (Christopher) (1991), A public management for all seasons?., Public Administration, vol. 69, primavera, pp. 3-19.

Hummel (Ralph) (1994), The Bureaucratic experience. A critique of life in the moder organization. New York, St. Martin's Press.

Ingraham (Patricia W.) (1993), of Pigs in Pokes and Policy Diffusion: Another Look at Pay-for-Performance, Public Administration Rev., vol. 53, n. ${ }^{\circ} 4$, julio-agosto, pp. 348-356.

JENKNSS (Bill) y GRAY (Andrew) (1994), ·Evaluación y el usuario: la experiencia del Reino Unido, Gestión y Análisis de Politicas Priblicas, n. ${ }^{\circ} 1$, septiembre-diciembre, pp. 47.57 (trad. de .Evaluating and the Consumer: The UK Experience, en J. MAYNE et al., Aduancing Public Policy Exaluation, Amsterdam, Elsevier Science, 1993, pp. 285-299).

LASHERAS (Miguel Ángel) y RUתz-HUERTa (Jesús) (1992), 'La gestión pública en el marco del proceso de convergencia europeo, Cuademos de Actualidad de Hacienda Priblica Española, n. ${ }^{\circ} 2 / 1992$, pp. 33-37.

LOPEZ-RUA (Joaquín) (1991), F Función pública galega., en Xaime Rodríguez-Arana, ed., Estudias sobre o Estatuto galego. $X$ aniversario da promulgación do Estatuto, Santiago de Compostela, Escola Galega de Administración Pública, pp. 399-412.

LOSADA (Carlos) y Xirau (Joan) (1995l, •Evaluación del desempeño. El plan de evaluación de recursos humanos de la Generalitat de Catalunya, Gestión y Análisis de Politicas Priblicas, n. ${ }^{\circ} 2$, enero-abril, pp. 67-77.

LuKE (Jeffrey S.) y CAIDEN (Gerald E.) (1989), .Coping with global interdependence, en James L. Perry, ed., Handbook of public Administration, San Francisco, Jossey Bass, pp. 83-93.

MArQuEz (Guillermo) (1995), La simplificación administrativa en el proceso de modernización de la Administración Pública. Comunicación a la •Xomada de estudio sobre simplificación administrativa. Santiago de Compostela, EGAP (mimeo).

MenXN (José L.) (1993), •Estado autonómico e Administracións públicas, en Xaime RODRIGUEZ-ARANA y Francisco J. SERNA, eds., Administración Unica. Relatorios das Xornadas celebradas en setembro de 1992 na EGAP. Santiago, EGAP, pp. 115-136.

MERTON (Roben K.) (1992), Teoria y estructura sociales, 3. ${ }^{2}$ edic., México, Fondo de Cultura Económica (trad. de Social Theory and Social Structure, 3. ${ }^{2}$ edic., New York, The Free Press, 1968). 
METCALF (Les) y Ruchards (Sue) (1989), La modemización de la gestión pública. Madrid, Ministerio para las Administraciones Públicas (trad. de Improving Public Mana gement, London, Sage, 1987).

March (James E.) y Oisev (Johan P.) (1983), Organizing Political Life: What Administrative Reorganizational Tell Us about Govemment, American Political Science Rev., vol. $\pi$, n. $^{\circ}$, junio, pp.281-2\%

Mattarelus (Bermardo G.) (1993), .la Codificazione del Dirito: Riflessione sull'esperienza fiancese contemporanea, Riv. Trimestrale di Dintto Pubblico, año XIIII, n. ${ }^{\circ} 4$, pp. 103 - 1061 .

Mises (Ludwig von) (1974), Burocracia. Madrid, Unión Edit (trad. de Bureaucracy, 2.' edic., Yale Univ. Press, 1962).

NiETo (Alejandro) (1989), Reforma administrativa y modemización de la Administración pública: e un problema pendiente?., en Inst. Vasco de Administración Pública, Modernización Administrativa, Oñati, pp. 101-115.

NiETo (Alejandro) (1992), .La reforma de la Administración pública., Rev. Vasca de Administración Priblica, n. $^{\circ}$ 34-11, septiembre-diciembre, pp. 145-157.

NIETO (Alejandro) (1990), -Las experiencias de modemización en España, Rev. Vasca de Administración Priblica, n." 45-1I, mayo-agosto, pp. 63-74.

NiSKANEv, Jr. (William A.) (1971), Bureaucracy and Representative Government. Chicago, Aldine Publ.

OLMEDA (José A.) (1988), Las Fuerzas Amadas en el Estado franquista: Panicipación politica, influencia presupuestaria..., Madrid, El Arquero.

ORTEGA (Luis) (1996), -La modemización de las Administraciones públicas,, Gestion y



OSBORNE (David) y GAEBLER (Ted) (1994), La reinvención del gobierno. Barcelona, Buenos Aires, Edic. Paidós (trad. de Reinuenting Government, Reading, Mass., Addison-Wesley Publ., 1992).

PAGE (Edward C.) (1992), Political Autbority and Bureaucratic Power, 2.' edic., London, Harvester-Wheatsheaf.

PETERS (B. Guy) (1991), The European bureaucrat: The applicability of Bureaucracy and representative govemment to non-American settings, en André Blais y Stéphane Dion, eds., The budget-maximizing bureaucrat, Pittsburgh, Univ. of Pittsburgh Press, pp. 303-353.

Peters (B. Guy) (1992), .Public Policy and Public Bureaucracy, en Douglas E. Ashford, ed., History and Context in Comparative Public Policy, Pittsburgh, Univ. of Pittsburgh Press, pp. 283-3 15.

Pouitt (Christopher) (1993), El gerencialismo y los servicios puiblicos (trad. española), Madrid, Instituto de Estudios Fiscales.

PORTER (Michael E.) (1991), La ventaja competitiva de las naciones (trad. española), Barcelona, Plaza y Janés.

PRECEDO (Andrés) (1993), -Las propuestas de división territorial en Galicia•, en Xosé R. Barrejro fervandez y Pablo Gonzalfz Mariñs, eds., Historia da Administración Piiblica, Santiago, EGAP, pp. 225.250.

QUERMONNE (Jean-Louis) (1985), -Les politiques institutionnelles: Essai d'interprétation et de typologie, en Madeleine GRA WnTZ y Jean LECA, eds., Traité de science politique. vol. 4, Les politiques publiques, Paris, PUF, pp. 61-88.

Ramio (Carles) y Subirats (Joan) (1990), •los aparatos administrativos de las Comunidades Autónomas (1980-1995): Entre el mimetismo y la diferenciación., Rev. Vasca de Administración Priblica. n. ${ }^{\circ}$ 45-11, mayo-agosto, pp. 151-181.

RICHARDS (Sue) (1992), Changing patterns of legitimation in public administration., Public Policy and Administration, vol. 7, n. ${ }^{\circ} 3$, invierno, pp. 15-28.

RICHARDS (Sue) (1994), •El paradigrna del cliente en la gestión pública (trad. de • Who defines the public good? The consumer paradigm in public management, Working paper, Public Nanagement Foundat, 1992), Gestion y Análisis de Politicas Priblicas, $\mathrm{n}^{\circ} 1$, septiembre-diciembre, pp. 5-16.

RIGBY (Darrell) (1993), . The secret history of process of reengineering, Plannino Rev., abril, pp. 24-27.

Rodriguez-ARava (Jaime) (1993), ed, O sector priblico económico galego. Santiago, Escola Galega de Administración Pública.

RodriGuez-ARAva (Jaime) (1997), ·Reforma administrativa y Estado autonómico en España, Reforma y Democracia, n. 7 , enero, pp. 157-194.

RodRigueZ-Arava (Jaime) y Agutar (Manuel) (1994), •Régimen de los organismos autónomos de la Comunidad Autónoma de Galiciar, en A. Pérez MORENo, ed., Administraciōn instrumental. Libro bomenaje a Manuel Francisco Clavero Arévalo, Madrid, Instituto Garcia Oviedo. Univ. Sevilla, Civitas, pp. 1673-1684.

RodriguEZ-ARAva (Jaime) y Bastos BouBETA (Miguel A.) (1996), Sobre la formación en el sector público Rev. Aragonesa de Administración Priblica, $n^{\circ} 8$, junio, pp. 235-252.

Rodrigutz RodricuEz (Dositeo) (1991), ‘A Administración pública galega, en Xaime Rodríguez-Arana, ed., Estudios sobre o Estatuto galego. $X$ aniversario da promulgación do Estatuto, Santiago de Compostela, Escola Galega de Administración Pública, pp. 391-398.

Rodriguez Rodriguez (Dositeo) (1994), •Principios inspiradores do Proxecto de Lei de modificación da Lei 4/1988, do 26 de maió da Función Pública de Galicia., en Mariano BaENa del ALCizar, ed., A reforma da Función Priblica galega. Relatorios da Xornada de estudio sobre o Proxecto..., Santiago, EGAP, pp. 11-22.

SERNA (Francisco J.) (1993), •Administración única para Galicia: unha proposta abelta•, en Xaime RODRiGUEz-ARANA y Francisco J. SERVA, eds., Administración Unica. Relatorios das Xomadas celebradas en setembro de 1992 na EGAP. Santiago, EGAP, pp. 27-42.

SERvA (Francisco J.) (1994), Os ordenamentos xurídicos autonómicos: problemática da súa configuración e integración, en Constantino AlVARELLOS, Francisco J. SERva y Guy Brabavt, Codificación normativa, Santiago de Compostela, Escola Galega de Administración Pública, pp. 41-61.

SUBIRATS (Joan) (1990), .Modernizing the Spanish public administration or reform in disguise, Barcelona, Inst. de Ciències Politiques i Socials (Working papers, $n .{ }^{n}$ 20).

SUBIRATs (Joan) y GOMA (Ricard) (1993), .Gestión pública y reforma administrativa en los 90: nuevos retos y oportunidades, en Izquierda Unida, Administraciones y Servicios Puiblicos. ¿Reforma o privatización? Madrid, pp. 63-71.

Trosa (Sylvie) (1992), •La modernisation est-elle evaluable?, Politiques et Management Public, vol. 10, n. 4, diciembre, pp. 65-83.

VÁzQuez ARES (1994), -Calidade total na Administración Pública e o Sistema de Xestión de Procedementos Administrativos, comunicación presentada al Seminario sobre Xestión de Calidade nas Administracións Públicas,, Santiago de Compostela, 1994 (mimeo).

VEBLEN (Thorstein) (1904), The Theory of Business Enterprise, New York, Charles SCHIBNERS SONS, citamos por reimpr. facsimilar, Clifton, Augustus M. Helley Publ. 1973.

VILAS Nogueira (J.) (1993), •A Administración pública no proceso estarutario de Galicia na II República, en Xosé R. Barreiro Fervivdoez y Pablo GonzÁlez Marnas, eds, Historia da Administración Pública, Santiago, EGAP, pp. 313-328.

VILoRu (Manuel) (1994), •El fenómeno modemizador en la Administración de la Comunidad de Madrid, Autonomies, n. ${ }^{\circ} 18$, pp. 147-172.

Vilıoru (Manuel) (1996), .La modernización de la Administración central en España, Revista Vasca de Administración Priblica, n." 45-11, mayo-agosto, pp. 95-115.

YeBra (Perfecto) (1993), •A Administración na doutrina galeguista, en Xosé R. BARRElro FeRvández y Pablo Gonź́lez MariNAs, eds., Historia da Administración Pública. Relatorios e comunicacións presentados..., Santiago, EGAP, pp. 281-312. 
